\title{
Colangitis esclerosante primaria: revisión de 12 años en dos centros de referencia
}

\author{
Ivonne 0 rellana $\mathbf{N}^{1}$, José Miguel Valera $\mathrm{M}^{1}$, Chun $\mathrm{Nei}^{1}{ }^{1}$, \\ Jaime Poniachik $\mathrm{T}^{1}$, Zoltán Berger $\mathrm{F}^{1}$, Ricardo Latorre $\mathrm{M}^{1}$, \\ G ladys Smok $S^{2}$, Javier Brahm B ${ }^{1,3}$. \\ Primary sclerosing cholangitis. A \\ twelve-year experience
}

Background: Primary sclerosing cholangitis (PSC) is a chronic cholestatic disorder characterized by progressive inflammation and fibrosis of the biliary tract, evolving to cirrhosis. It is commonly associated with inflammatory bowel disease (IBD). Aim: To communicate the clinical characteristics of patient with PSC seen in two reference centers. Patients and methods: Review of medical records of patients with PSC confirmed by liver biopsies. The clinical picture, laboratory abnormalities, imaging studies and treatment were recorded. Results: Thirty three patients (aged 16 to 73 years, $64 \%$ female) were identified. They corresponded to $1.7 \%$ of liver biopsies done between 1991-2003. Clinical presentation was a cholestatic picture in $40 \%$, right upper abdominal pain in 36\%, a dysenteric syndrome in $9 \%$ and varied symptoms in 15\%. Laboratory tests showed cholestasis in $94 \%$ and positive anti ANCA, SMA, ANA and AMA antibodies in 28, 18, 15 and 9\% of cases, respectively. Endoscopic retrograde cholangiopancreatography and magnetic resonance cholangiography were diagnostic in 43 and $58 \%$ of patients, respectively. There was an association with ulcerative colitis in $12 \%$ of cases. Liver biopsies showed grade I PSC in $76 \%$ and grade II-III in $6 \%$ of patients. It also showed a concomitant chronic hepatitis and primary biliary cirrhosis in 12 and $6 \%$ of cases, respectively. Treatment consisted on ursodeoxycholic acid (UDCA) in 45\%, UDCA plus 5-aminosalicylic acid derivatives in $12 \%$ and UDCA plus immunosuppresors in $12 \%$ of patients. Two patients had to be transplanted. Conclusions: PSC is an uncommon cause of chronic liver disease. It is suspected in cholestatic patients and confirmed with a liver biopsy. It can be associated with other autoimmune hepatic and extrahepatic diseases (Rev Méd Chile 2005; 133: 776-80).

(Key Words: Cholangitis, sclerosing; Cholestasis, intraheaptic; Hepatitis, autoimmune; Ursodeoxycholic acid)

Recibido el 30 de julio, 2004. Aceptado el 10 de mayo, 2005.

${ }^{1}$ Sección de Gastroenterología y ${ }^{2}$ Departamento de Anatomía Patológica, Hospital Clínico de la Universidad de Chile, ${ }^{3}$ Departamento de Gastroenterología, Clínica Las Condes. Santiago de Chile.

Correspondencia a: Dr. Javier Brahm B. Sección de Gastroenterología, Hospital Clínico Universidad de Chile. Santos Dumont 999, Independencia. Teléfono: 9788350. Fax: 9788349. E-mail: jbrahm@redclinica.cl 
L a colangitis esclerosante primaria (CEP) es una enfermedad hepática colestásica crónica, progresiva y de mal pronóstico, que se asocia hasta en $70 \%$ con una enfermedad inflamatoria intestinal (EII) y que tiene un riesgo elevado de desarrollar un colangiocarcinoma. Si bien su etiología es desconocida, se produce inflamación y fibrosis progresiva de la vía biliar intra y extrahepática, evolucionando a una cirrosis biliar e insuficiencia hepática secundaria ${ }^{1,2}$.

El diagnóstico de CEP suele basarse en una combinación de antecedentes clínicos y alteraciones bioquímicas, radiológicas e histológicas. Una vez establecido éste, se han utilizado algunas alternativas de tratamiento médico, sin los resultados esperados, tanto en sobrevida como en mortalidad, siendo actualmente el trasplante hepático la única terapia para los pacientes en estadios avanzados o con complicaciones graves.

Por ser la CEP una enfermedad poco frecuente en nuestro medio, se realizó este trabajo con el objetivo de evaluar sus características clínicas.

\section{PACIENTES y MÉTOdOS}

Se estudiaron retrospectivamente los pacientes con diagnóstico histológico de CEP, a partir de las biopsias hepáticas percutáneas realizadas en el Hospital Clínico de la Universidad de Chile y en Clínica Las Condes, entre los años 1991 y 2003. Se revisaron las fichas clínicas de estos pacientes, registrándose las características clínicas, alteraciones de laboratorio, enfermedades asociadas, estudio de imágenes y tratamiento. El análisis y revisión histológica fue realizada por uno de los coautores (GS), quien evaluó el estadio de la enfermedad según los criterios de Ludwig et al: estadio I, aumento de tejido conectivo con ensanchamiento de los espacios porta; estadio II, aumento del tejido conectivo en la zona periportal e inflamación mínima; estadio III, formación de septos fibrosos en el parénquima hepático y estadio IV, cirrosis $^{3}$.

\section{Resultados}

La población estudiada corresponde a 33 de un total de $1.955(1,7 \%)$ pacientes biopsiados con distintas indicaciones en los últimos 12 años, con una edad promedio de 45 años (rango 16 a 73 años), siendo $64 \%$ de sexo femenino.

La presentación clínica más predominante fue un síndrome colestásico con ictericia, coluria y prurito en $40 \%$ de los casos, seguido por astenia, dolor en hipocondrio derecho en 36\%, síndrome disentérico en $9 \%$ y polisintomáticos en $15 \%$. Alteraciones de las pruebas hepáticas con colestasis (fosfatasa alcalina elevada entre 2 y 16 veces y GGT entre 2 y 26 veces) se observó en $94 \%$ y acompañado de elevación de transaminasas (2 a 20 veces) en $45 \%$ (Tabla 1 ).

Dentro de los marcadores inmunológicos, los anticuerpos anti-citoplasma de neutrófilos (ANCA) fueron positivos en $28 \%$, los anti-músculo liso (AML) en 18\% (títulos entre $1 / 40$ y $1 / 160$ ), los antinucleares (ANA) en 15\% (títulos entre 1/40 y 1/320) y anticuerpos anti-mitocondriales (AMA) en el $9 \%$ (títulos entre $1 / 40$ y 1/320), de los casos solicitados. La cuantificación de IgG estuvo elevada sólo en 15\% (rango entre 2.300 y 4.720) (Tabla 2).

En 15 de los 33 pacientes, los métodos de imágenes apoyaron el diagnóstico de CEP, destacando los hallazgos encontrados en la colangiopancreatografía endoscópica retrógrada en 3 de 7 casos (43\%), en la colangiografía por resonancia magnética en 10 de 17 casos (58\%) y en la ecografía abdominal en 2 de los 33 casos (6\%) en que se realizaron (Tabla 2 ).

Respecto a la asociación entre CEP y EII, sólo en 14 de los 33 pacientes (42\%) se realizó una colonoscopia, siendo compatible con colitis ulcerosa idiopática (CUI) en 4 de ellos (28\%), todos con

\section{Tabla 1. Presentación clínica y de laboratorio en 33 pacientes con colangitis esclerosante primaria}

\begin{tabular}{|lrr|}
\hline Clínica y laboratorio & $\mathrm{n}$ & $\%$ \\
\hline Ictericia, coluria y prurito & 13 & 40 \\
Astenia y dolor hipocondrio derecho & 12 & 36 \\
Síndrome disentérico & 3 & 9 \\
Polisintomáticos & 5 & 15 \\
$\quad$ (mezcla de todos los anteriores) & & \\
Alteración pruebas hepáticas & & \\
$\quad$ Colestasis & 31 & 94 \\
$\quad$ Patrón mixto & 15 & 45 \\
\hline
\end{tabular}


Tabla 2. Resultados de exámenes inmunológicos y de imágenes en 33 pacientes con colangitis esclerosante primaria

\begin{tabular}{|lrrrr|}
\hline Exámenes & Positivos / & Realizados / & $(\%)$ \\
\hline AML & 6 & $/$ & 33 & $(18)$ \\
ANA & 5 & $/$ & 33 & $(15)$ \\
AMA & 3 & $/$ & 33 & $(9)$ \\
ANCA & 2 & $/$ & 7 & $(28)$ \\
IgG elevada & 5 & $/$ & 33 & $(15)$ \\
CPER & 3 & $/$ & 7 & $(43)$ \\
Colangio RM & 10 & $/$ & 17 & $(58)$ \\
Ecografía abdominal & 2 & $/$ & 33 & $(6)$ \\
\hline
\end{tabular}

AML: anti músculo liso. ANA: antinucleares. AMA: anti mitocondriales. ANCA: anti citoplasma de neutrófilos. CPER: colangiopancreatografía endoscópica retrógrada. CRM: colangiografía por resonancia magnética y ecografía abdominal.

confirmación histológica. Dos pacientes debutaron con CUI y desarrollaron posteriormente una CEP.

La biopsia hepática que determinó el diagnóstico mostró estadio I de la enfermedad en 25 casos (76\%) y estadio II-III en 2 casos (6\%). Además, se asoció con hepatitis autoinmune (HAI) en 4 casos $(12 \%)$ y con cirrosis biliar primaria (CBP) en 2 casos (6\%) (Tabla 3).

Cuatro pacientes que presentaron inicialmente el cuadro clínico e histológico de una HAI, tuvieron en su evolución una inadecuada respuesta a los inmunosupresores y desarrollaron colestasis, siendo las nuevas biopsias hepáticas compatibles con CEP.

El tratamiento que recibieron los pacientes incluyó ácido ursodeoxicólico (AUDC) en dosis de 500 a $1.500 \mathrm{mg} /$ día en 15 casos (45\%), AUDC asociado a derivados 5 ASA en 4 casos (12\%) y AUDC más inmunosupresores (prednisona entre 7,5 y $50 \mathrm{mg} /$ día, azatioprina entre 50 y $100 \mathrm{mg} /$ día 0 ambos) en los 4 casos asociados a HAI (12\%). Los otros 10 pacientes (30\%) no recibieron AUDC por problemas económicos. Dentro de la evolución, 4 pacientes (12\%) fueron sometidos a colecistectomía y un paciente presentó abscesos hepáticos que fueron tratados con antibióticos intravenosos, drenaje y repetidas dilataciones de la vía biliar.
Tabla 3. H istología en 33 pacientes con colangitis esclerosante primaria (CEP) y asociación con otras enfermedades hepáticas

\begin{tabular}{|lcr|}
\hline Biopsia - Estado & Pacientes & / \\
\hline CEP estadio I & 25 & $(76)$ \\
CEP estadio II-III & 2 & $(6)$ \\
HAI a CEP & 4 & $(12)$ \\
CEP a CBP & 2 & $(6)$ \\
& 33 & $(100)$ \\
\hline
\end{tabular}

HAI: hepatitis autoinmune. CBP: cirmosis biliar primaria.

Dos pacientes (6\%) fueron sometidos a trasplante hepático por presentar complicaciones como hemorragia digestiva alta por várices $\mathrm{y}$ peritonitis bacteriana espontánea con deterioro del score de Child-Pugh B-C, uno de los cuales falleció por rechazo crónico, complicado con estenosis de la anastomosis de vía biliar y sepsis no controlada.

\section{DisCUSIÓN}

Este estudio retrospectivo y descriptivo confirma que la CEP es una enfermedad poco frecuente en nuestro medio, encontrándose sólo 33 casos $(1,7 \%$ de las 1.955 biopsias hepáticas realizadas por hepatopatía crónica) en dos centros de referencia en 12 años. Lo anterior, a pesar de ser una patología mejor descrita en los últimos años y del progreso en las técnicas de imágenes para plantear o precisar su diagnóstico ${ }^{1-3}$.

Es importante considerar que, si bien todos los pacientes tenían alteraciones colestásicas de laboratorio, menos de la mitad (40\%) tenía síntomas o signos como ictericia, coluria o prurito. Tal como la literatura lo describe, se requiere por lo tanto un alto índice de sospecha para pesquisar esta patología en pacientes asintomáticos con estas alteraciones, destacando el estudio con imágenes y la histología para precisar el diagnóstico ${ }^{1,4}$. En este sentido, exámenes de gran utilidad son los que permiten visualizar las anormalidades en la vía biliar intra y extrahepática descritas en la CEP, siendo previamente la colangiopancreatografía endoscópica retrógrada ${ }^{5}$ y actualmente la colan- 
giografía por resonancia magnética ${ }^{6}$ de primera elección, por tratarse de un examen no invasivo y de alta resolución. Este proporciona información de los ductos biliares y del tejido periductal y permite descartar neoplasias, compromiso hepático y de las estructuras vecinas, en caso de necesidad de trasplante hepático ${ }^{7}$. En efecto, este examen confirmó el diagnóstico en $58 \%$ de los casos de nuestra serie en que se efectuó (10 de 17 pacientes).

En 55\% de los pacientes las técnicas de imagen no mostraron alteraciones. Estos casos corresponderían a una variedad de CEP que compromete solamente los pequeños conductos, siendo por ello la biopsia hepática, junto con la clínica y laboratorio, las que nos permiten hacer el diagnóstico.

Por otra parte, la biopsia hepática es necesaria para confirmar el diagnóstico de CEP y tiene, además, valor pronóstico, al establecer el estadio de la enfermedad, determinando la inflamación y daño ductal, junto con la presencia de fibrosis y cirrosis $^{5}$. En el presente trabajo, al momento del diagnóstico, $76 \%$ de los pacientes estaban en un estadio inicial y sólo $6 \%$ en estadio II o III.

Existe una variedad de enfermedades autoinmunes asociadas a CEP, particularmente las EII. Aunque los primeros estudios sugirieron una asociación de 25 a 30\% de EII, estudios más recientes indican que estaría presente entre $75 \mathrm{y}$ 85\% de los pacientes con CEP, particularmente la $\mathrm{CUI}^{3}$. En nuestro estudio encontramos una baja frecuencia de CUI ( $28 \%$ de los casos con colonoscopia realizada), debido probablemente al pequeño número de exámenes efectuados, a la mayor frecuencia de CEP en estadio inicial (por lo que se debe reevaluar en su evolución la aparición de EII) y la eventual normalidad del examen endoscópico en pacientes que utilizaban esteroides o inmunosupresores previo a este examen. Dentro de los casos que tenían ambas enfermedades, sólo algunos pacientes presentaron inicialmente síntomas de CUI antes de desarrollar CEP (6\%), mientras la mayoría se manifestó inicialmente sólo como una CEP (94\%).

Otras enfermedades descritas en menor frecuencia asociadas a la CEP son la HAI y la CBP, lo que observamos 4 y 2 pacientes, respectivamente. En las cuatro pacientes que presentaron inicial- mente HAI y que evolucionaron con un perfil colestásico y mala respuesta a inmunosupresores, se realizó una nueva biopsia hepática que confirmó CEP junto con alteraciones compatibles en las imágenes, como ya se describió previamente ${ }^{8}$. Aunque existe una considerable sobreposición de las manifestaciones clínicas, bioquímicas e histológicas de la CEP y la CBP, suele ser posible distinguir estas enfermedades con certeza. Para esto debemos basarnos en la especificidad de los anticuerpos AMA fracción M2 para CBP, el compromiso de la vía biliar extrahepática en la CEP y las características histológicas informadas por un patólogo experto.

La bien descrita asociación de CEP con enfermedades autoinmunes, refuerza su posible naturaleza inmunológica, teniendo fuerte relación con el sistema HLA y la presencia de autoanticuerpos: AML en 11 a 15\%, ANA en 35\% y ANCA en 60\% ${ }^{9}$, siendo en nuestro estudio positivos en 18\%, 15\% y $28 \%$, respectivamente ${ }^{10,11}$.

Dentro de las complicaciones de la CEP están la colangitis recidivante, litiasis de la vía biliar, adenocarcinoma de vías biliares y la cirrosis ${ }^{6,12}$. En este estudio, se encontró que 4 pacientes (12\%) fueron sometidos a colecistectomía por presencia de litiasis y sólo un caso (3\%) presentó estenosis de la anastomosis y se complicó con abscesos hepáticos, que requirió varias dilataciones. Otro caso que tenía un colédoco fino y fibrótico, evolucionó satisfactoriamente.

Dos pacientes presentaron cirrosis hepática descompensada, con deterioro del score de ChildPugh y complicaciones propias de la cirrosis como hemorragia digestiva y peritonitis bacteriana espontánea, lo que motivó el trasplante hepáti$\mathrm{co}^{13}$. Uno de ellos se complicó y falleció por rechazo crónico y complicaciones estenóticas de la vía biliar y sepsis no controlada. No encontramos pacientes con colangiocarcinoma, probablemente debido a las limitaciones propias de un estudio retrospectivo.

Respecto al tratamiento de la CEP, se recomiendan medidas para aliviar la ictericia y el prurito, así como también el reemplazo de vitaminas liposolubles. Los tratamientos con inmunosupresores $^{14}$ no han demostrado valor, y si bien el uso del AUDC en dosis mayores a $20 \mathrm{mg} / \mathrm{kg}$ mejora los parámetros bioquímicos y el prurito, 
no ha demostrado claramente un beneficio en la progresión, complicaciones, sobrevida o necesidad de trasplante ${ }^{15,16}$. En este sentido, cerca de $70 \%$ de nuestros pacientes se trató con AUDC y alrededor de 30\% no tenían tratamiento farmacológico por limitaciones económicas.

En suma, la CEP es una causa poco frecuente de enfermedad hepática crónica en adultos en nuestro medio. Tiene síntomas propios, alteracio-

\section{REFERENCIAS}

1. Lee YM, Kaplan M. Primary sclerosing cholangitis. N Engl J Med 1995; 332: 924-33.

2. Porayko MK, Wiesner RH, La Russo NF, Ludwig J, Mac Carty RL, Steiner BL et al. Patients with asymptomatic sclerosing cholangitis frequently have progressive disease. Gastroenterology 1990; 98: 1594-602.

3. Ludwig J, Barham S, La Russo NF, Elveback LR, WIESNER RH, McCAL JT. Morphologic features of chronic hepatitis associated with primary sclerosing cholangitis or chronic ulcerative colitis. Hepatology 1981; 1: 632-40.

4. Angulo P, LiNdor KD. Primary sclerosing cholangitis. Hepatology 1999; 30: 325-32.

5. Ludwig J, La Russo NF, WiesNer RH. Primary sclerosing cholangitis. In Peters RL and Craig JR, eds. Liver Pathology, Contemporary Issues in Pathology. New York: Churchill Livingstone 1986; 193-214.

6. Massom A, Bret $H$, Bret P. The role of magnetic resonance cholangiography in primary sclerosing cholangitis. J Hepatol 2000; 33: 659-60.

7. Ramage JK, Donaghy A, Farrant JM, Iorns $R$, WiшAMs R. Serum tumor markers for the diagnosis of cholangiocarcinoma in primary sclerosing cholangitis. Gastroenterology 1995; 108: 865-9.

8. Vaiera JM, Smok G, Fernández M, Brahm J. Evolución de hepatitis autoinmune a colangitis esclerosante primaria. Gastroent Latinoam 2002; 13: 45-50. nes bioquímicas de colestasis y biopsia hepática característica. Además, se puede asociar a otras enfermedades autoinmunes como HAI, CBP y EII. Dentro de las complicaciones a considerar en su presentación o evolución están la colangitis y el colangiocarcinoma, las que deben ser investigadas. Finalmente, el trasplante hepático sigue siendo la única alternativa terapéutica definitiva en casos muy seleccionados.

9. Angulo P, Peter JB, Gershwin E, DeSotel C, SHOENFELD Y, LINDOR KD. Serum autoantibodies in patients with primary sclemsing cholangitis. J Hepatol 2000; 32: 182-7.

10. Wiesner RH, La Russo NF, Ludwig J, Dickson ER. Comparison of the clinicopathologic features of primary sclerosing cholangitis and primary biliary cirrhosis. Gastroenterology 1985; 88: 108-14.

11. LiNDOR KD, WiesNer RH, La Russo NF, Dickson ER. Chronic active hepatitis: overlap with primary biliary cirrrhosis and primary sclerosing cholangitis. In Czaja AJ and Dickson ER, (eds.) Chronic Active Hepatitis: The Mayo Clinic Experience. New York: Marcel Dekker, Inc, 1986; 171-87.

12. La Russo NF, WiesNeR RH, LUdwig J. Colangitis esclerosante primaria. Tratado de Hepatología Clínica, Tomo II, Ediciones Científicas y Técnicas, Editorial Masson-Salvat, Barcelona, 1993; 893-902.

13. BJoRo K, SchrumpF E. Liver transplantation for primary sclerosing cholangitis. J Hepatol 2004; 40: 570-2.

14. Glucocorticosteroids for primary sclerosing cholangitis. Cochrane Database Syst Rev 2004, CD 004036 .

15. Bile acids for primary sclerosing cholangitis. Cochrane Database Syst Rev 2003, CD003626.

16. Lindor KD for the Mayo Primary Sclerosing Cholangitis-Ursodeoxycholic Acid Study Group. Ursodiol for primary sclerosing cholangitis. N Engl J Med 1997; 336: 691-5. 